\title{
Elliptic Dunkl operators, root systems, and functional equations
}

\author{
V. M. Buchstaber*, G. Felder** and A. P. Veselov*
}

March 1994

\author{
*Department of Mathematics and Mechanics, Moscow State University, \\ 119899 Moscow, Russia \\ **Department of Mathematics, University of North Carolina at Chapel Hill, \\ Chapel Hill, NC 27599-3250, USA
}

\section{Introduction}

In the paper [国, Dunkl introduced the following difference-differential operators acting on functions on a Euclidean space $V$, related to arbitrary finite groups $G$ generated by orthogonal reflections in $V$ :

$$
\nabla_{\xi}=\partial_{\xi}+\sum_{\alpha \in R_{+}} k_{\alpha}(\alpha, \xi) \frac{1}{(\alpha, x)} \hat{s}_{\alpha}
$$

Here $\partial_{\xi}$ denotes the partial derivative in direction $\xi \in V, R$ is the root system of the group $G$, i.e., the set of unit normals to the reflection hyperplanes, $R_{+}$ is its positive part with respect to some generic linear form on $V, k_{\alpha}=k(\alpha)$ is a $G$-invariant function on $R, s_{\alpha}$ is the reflection corresponding to the root $\alpha \in R$, and $\hat{s}_{\alpha}$ is the operator on the space of functions on $V$ :

$$
\hat{s}_{\alpha} f(x)=f\left(s_{\alpha}(x)\right) .
$$

To be precise, Dunkl used slightly different operators, which are conjugated to (1) by the operator of multiplication by $\prod(\alpha, x)^{k_{\alpha}}$.

The main property of the Dunkl operators is given by the following 
Theorem 1 (Dunkl) The operators (1) commute with each other:

$$
\left[\nabla_{\xi}, \nabla_{\eta}\right]=0
$$

for all $\xi, \eta \in V$.

The goal of this work is to describe certain generalizations of the Dunkl operators (四), preserving the property (2). Some of these results were announced in [2].

In Section 1 we consider generalizations of the form

$$
\nabla_{\xi}=\partial_{\xi}+\sum_{\alpha \in A_{+}} k_{\alpha}(\alpha, \xi) \frac{1}{(\alpha, x)} \hat{s}_{\alpha},
$$

where $A_{+}$is the set of unit normals to some set $S$ of hyperplanes in $V$ passing through the origin, $A_{+}$is its positive part, and $k_{\alpha}=k(\alpha)$ is some function on $A_{+}$. We show that the commutativity of the operators $\nabla_{\xi}$ implies that $S$ is the set of reflection hyperplanes of some Coxeter group $G, A_{+}=R_{+}$, and $k$ is $G$-invariant.

In the Section 2, we consider operators of the form

$$
\nabla_{\xi}=\partial_{\xi}+\sum_{\alpha \in R_{+}}(\alpha, \xi) f_{\alpha}((\alpha, x)) \hat{s}_{\alpha},
$$

where $f_{\alpha}(z)$ are functions of one variable, not identically 0 .

The commutation relations (2) are equivalent to a system of functional equations for the functions $f_{\alpha}, \alpha \in R_{+}$. In the case when $G$ is the Weyl group $W$ of a simple Lie algebra, we show that, with the exception of $A_{1}$, $B_{2}$, the only $W$-invariant solutions, i.e., such that

$$
\hat{s}_{\alpha} \nabla_{\xi}=\nabla_{s_{\alpha}(\xi)} \hat{s}_{\alpha},
$$

for all $\alpha \in R_{+}$, are Dunkl's solutions, in accordance with [3].

While the $A_{1}$ case is trivial, the $B_{2}$ case leads to the classical theory of Landen's and Jacobi's transformations of elliptic functions. We give the general $W$-invariant solution in this case. This result has an interesting topological application in the theory of elliptic genera (see [4]).

In Section 3, we give a solution of the functional equations in terms of elliptic functions, for arbitrary reduced root systems. This solution gives 
families of commuting differential-difference operators that we call elliptic Dunkl operators.

We then show in Section 4, using techniques from [0], [6], that these are essentially all solutions in the $A_{n-1}$ case.

In Section 5, quantum elliptic Dunkl operators are introduced. These are pairwise commutative families of difference operators. They depend on a parameter $\mu$, and are such that elliptic Dunkl operators appear in the first order term (semiclassical approximation) of their expansion in power of $\mu$. These operators are related to the transfer matrices associated with the $R$-matrix of [7], [8].

We conclude by discussing the possible applications of our results to the theory of integrable $n$-body systems.

\section{Operators of Dunkl type and Coxeter groups}

Let $S$ be a finite set of hyperplanes in a Euclidean space $V$ passing through the origin, $A$ the set of unit normals to the hyperplanes in $S$ (two for each hyperplane), $A_{+}$the positive half of $A$ with respect to some linear form on $V$, and $k_{\alpha}$ some non-zero coefficients.

Let $\nabla_{\xi}$ be the operator

$$
\nabla_{\xi}=\partial_{\xi}+\sum_{\alpha \in A_{+}} k_{\alpha}(\alpha, \xi) \frac{1}{(\alpha, x)} \hat{s}_{\alpha}, \quad k_{\alpha} \neq 0 .
$$

Theorem 2 The operator $\nabla_{\xi}$ and $\nabla_{\eta}$ commute for arbitrary $\xi$ and $\eta \in V$ if and only if $A_{+}$coincides with $R_{+}$for some Coxeter group $G$ and $k(\alpha)=k_{\alpha}$ is a $G$-invariant function.

Proof: The commutator $\left[\nabla_{\xi}, \nabla_{\eta}\right]$ can be rewritten in the form I+II+III (see [9]), where

$$
\begin{aligned}
\mathrm{I} & =\left[\partial_{\xi}, \partial_{\eta}\right]=0, \\
\mathrm{II} & =\partial_{\xi}\left(\sum_{\alpha} k_{\alpha} \frac{(\alpha, \eta)}{(\alpha, x)} \hat{s}_{\alpha}\right)-\partial_{\eta}\left(\sum_{\alpha} k_{\alpha} \frac{(\alpha, \xi)}{(\alpha, x)} \hat{s}_{\alpha}\right)=0, \\
\mathrm{III} & =\sum_{\alpha, \beta \in A_{+}} k_{\alpha} k_{\beta}\langle\alpha, \beta\rangle_{\xi, \eta} \frac{1}{(\alpha, x)\left(s_{\alpha}(\beta), x\right)} \hat{s}_{\alpha} \hat{s}_{\beta},
\end{aligned}
$$


where $\langle\alpha, \beta\rangle_{\xi, \eta}=(\alpha, \xi)(\beta, \eta)-(\alpha, \eta)(\beta, \xi)$. One sees that to have a cancellation of the terms in the sum corresponding to fixed rotation $s_{\alpha} s_{\beta}$, it is necessary that, together with $\alpha, \beta$, the vector $\gamma=s_{\alpha}(\beta)$, also belongs to $A$. This means that $A$ is the root system $R$ for some Coxeter group.

To prove that $k$ is $G$-invariant, let us recall that if it is so, then $\left[\nabla_{\xi}, \nabla_{\eta}\right]=$ 0 according to Dunkl's theorem. Suppose that $k$ is not invariant, i.e., there exist two roots $\alpha, \beta$ such that $k_{\beta} \neq k_{\gamma}, \gamma=s_{\alpha}(\beta)$. Subtracting from III the Dunkl identity with appropriate coefficients, gives a relation of the form

$$
\sum_{\alpha, \beta \in A_{+}} c_{\alpha, \beta}\langle\alpha, \beta\rangle_{\xi, \eta} \frac{1}{(\alpha, x)\left(s_{\alpha}(\beta), x\right)} \hat{s}_{\alpha} \hat{s}_{\beta}=0
$$

where $c_{\alpha, \beta}=0, c_{\gamma, \alpha} \neq 0$. But this is impossible, since the pole at $(\gamma, x)=0$ cannot be canceled.

Thus we have shown that Coxeter groups arise naturally as a commutativity condition for a natural generalization of Dunkl operators.

\section{Functional equations for the coefficients of generalized Dunkl operators}

Let us now consider for given Coxeter group $G$ the following generalizations of Dunkl's operators:

$$
\nabla_{\xi}=\partial_{\xi}+\sum_{\alpha \in R_{+}}(\alpha, \xi) f_{\alpha}((\alpha, x)) \hat{s}_{\alpha}
$$

It is convenient to extend the definition of $f_{\alpha}$ to all $\alpha \in R$, by setting

$$
f_{-\alpha}(z)=-f_{\alpha}(-z) \text {. }
$$

With this definition, $\nabla_{\xi}$ is independent of the choice of the positive part $R_{+}$ of $R$. In fact, we can replace $R_{+}$by any subset of $R$ consisting of one normal vector for each hyperplane, without changing $\nabla_{\xi}$.

We may choose as before $R$ to consist of unit vectors, but, since Weyl groups will be considered later, it is convenient to allow normal vectors to have arbitrary length, still preserving the condition that we have two normal vectors $\alpha$ and $-\alpha$ for each hyperplane in $S$. At this point, this is no generalization, since the operators do not change if we replace $\alpha$ by a multiple $\alpha^{\prime}=c \alpha$ and replace the corresponding function $f_{\alpha}$ by the function $f_{\alpha^{\prime}}(z)=c^{-1} f_{\alpha}\left(c^{-1} z\right)$. 
A calculation analogous to the previous one leads to the following formula

$$
\left[\nabla_{\xi}, \nabla_{\eta}\right]=\sum_{\alpha, \beta \in R_{+}}\langle\alpha, \beta\rangle_{\xi, \eta} f_{\alpha}((\alpha, x)) f_{\beta}\left(\left(s_{\alpha}(\beta), x\right)\right) \hat{s}_{\alpha} \hat{s}_{\beta}
$$

where, as above, $\langle\alpha, \beta\rangle_{\xi, \eta}=(\alpha, \xi)(\beta, \eta)-(\alpha, \eta)(\beta, \xi)$.

Theorem 3 The commutativity condition for the operators (6)

$$
\left[\nabla_{\xi}, \nabla_{\eta}\right]
$$

is equivalent to the system of functional equations

$$
\sum_{\alpha, \beta \in R_{+}: s_{\alpha} s_{\beta}=r}\langle\alpha, \beta\rangle f_{\alpha}((\alpha, x)) f_{\beta}\left(\left(s_{\alpha}(\beta), x\right)\right)=0,
$$

for any given rotation $r$.

Here $\langle\alpha, \beta\rangle$ denotes the oriented area of the parallelogram with sides $\alpha, \beta$, with respect to some orientation of the plane spanned by these two vectors, which is perpendicular to the rotation axis of $r$. Obviously, the equation (9) is independent of the choice of orientation.

Example: For the root system of type $A_{2}$ (see Fig. 1), we have the following functional equation for the three functions associated with the three roots labeled in Fig. 1. The functions associated to the other roots are then determined by (7).

$$
f(x-y) g(x-z)+g(y-z) h(y-x)+h(z-x) f(z-y)=0 .
$$

Let us now discuss $G$-invariant solutions of the functional equations (10): $f_{g(\alpha)}=f_{\alpha}$ for all $g \in G$, or, equivalently,

$$
\hat{g} \nabla_{\xi}=\nabla_{g(\xi)} \hat{g}, \text { for all } g \in G .
$$

We assume that none of the functions $f_{\alpha}$ vanishes identically. We restrict ourselves to the case where $G$ is the Weyl group $W$ a semisimple Lie algebra. Obviously it is sufficient to consider the case of a simple Lie algebra. Excluding the one dimensional case $A_{1}$ where commutativity does not give any restriction on $f_{\alpha}$, the first case we consider is the case $A_{n-1}$, where $G=W$ is the symmetric group $S_{n}$. 


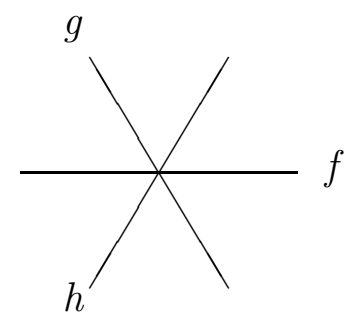

Figure 1: The root system of type $A_{2}$

Proposition 4 For root systems of type $A_{n-1}, n \geq 3$, the only $S_{n}$-invariant solution of (9) meromorphic in a neighborhood of the origin is

$$
f_{\alpha}(z)=\frac{C}{z}
$$

which corresponds to the usual Dunkl operators.

Proof: Consider the functional equation (9). For given $r$, it involves only roots in the 2-plane orthogonal to the rotation axis of $r$. This plane is spanned by any two roots $\alpha, \beta$ with $s_{\alpha} s_{\beta}=r$.

In the $A_{n-1}$ case, the roots in any such plane build a root system of type $A_{1} \times A_{1}$ or $A_{2}$. In the former case roots are orthogonal and (9) is identically satisfied for all $f$. It is therefore sufficient to consider the case $n-1=2$. Let us rewrite (10) in the form

$$
f(u) g(u+v)+g(v) h(-u)+h(-u-v) f(-v)=0 .
$$

$S_{3}$-invariant solutions correspond to $f=g=h$, where $f$ is an odd function, since $f_{\alpha}(z)=f_{-\alpha}(z)=-f_{\alpha}(-z)$. This leads to the following equation for $f$ :

$$
f(u) f(u+v)-f(v) f(u)+f(u+v) f(v)=0 .
$$

If $f$ vanishes at a point $u$, then $f(v) f(u+v)=0$ for all $v$, and $f$ vanishes identically. If $f$ does not vanish anywhere, we get, after substitution $\phi=1 / f$,

$$
\phi(u+v)=\phi(u)+\phi(v),
$$


which has the only solution $\phi(z)=c z$.

Let us consider some more examples of low rank. It will be shown below that these examples essentially cover the whole theory.

Example: In the case of $G_{2}$ (see Fig. 2), one has two $A_{2}$ systems. Hence the the invariant solutions have the form

$$
f(z)=\frac{A}{z}, \quad g(z)=\frac{B}{z},
$$

for arbitrary $A$ and $B$.

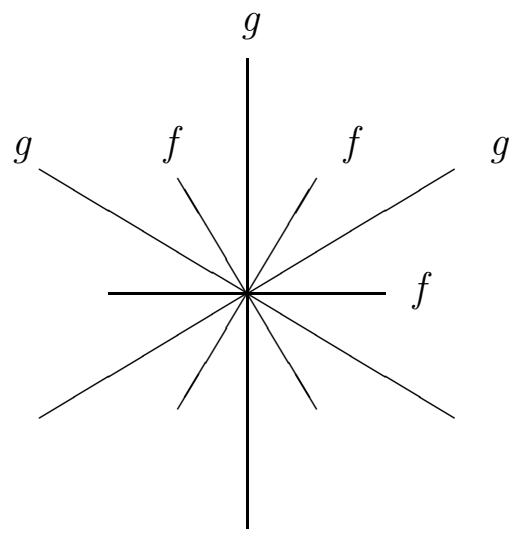

Figure 2: The root system of type $G_{2}$

Example: In the $B_{2}$ case (see Fig. 3) one has the following functional equation for the symmetric solution:

$$
f(x)(g(x+y)+g(x-y))+f(y)(g(x+y)-g(x-y))=0,
$$

where $f$ and $g$ are odd functions. In this case we have more complicated solutions, such as

$$
f(z)=\cot (z) \quad g(z)=\frac{1}{\sin (z)} .
$$

The general solution will be given below. 


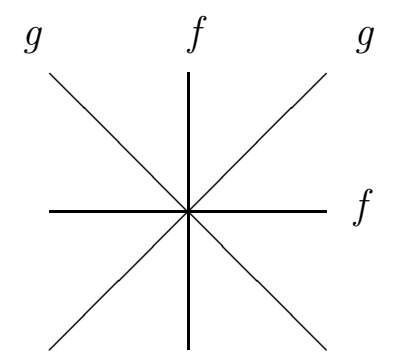

Figure 3: The root system of type $B_{2}$

Example: In the $B_{3}$ case, $R=\left\{ \pm e_{i}, \pm e_{i} \pm e_{j}\right\}$, where $\left\{e_{1}, e_{2}, e_{3}\right\}$ is an orthonormal basis of $V$. Let us find the most general invariant solution. The $\operatorname{roots} \alpha=e_{1}+e_{2}$, and $\beta=e_{2}+e_{3}$ form a subsystem isomorphic to $A_{2}$. Thus $f_{\alpha}(z)=f_{\beta}(z)=C / z$. Now consider the $B_{2}$ system generated by $\gamma=e_{1}$ and $\alpha$. So we have to solve the functional equation (12) with $g=C / z$ :

$$
f_{\gamma}(x)\left(\frac{C}{x+y}+\frac{C}{x-y}\right)+f_{\gamma}(y)\left(\frac{C}{x+y}-\frac{C}{x-y}\right)=0,
$$

or, equivalently (if $C \neq 0$ ),

$$
x f_{\gamma}(x)-y f_{\gamma}(y)=0
$$

which implies $f_{\gamma}(z)=D / z$. It follows that the most general invariant solution is Dunkl's solution.

Theorem 5 For all root systems of simple Lie algebras except $A_{1}, B_{2}$, all $W$-invariant solutions of the functional equation (9) have the form

$$
f_{\alpha}(z)=\frac{k_{\alpha}}{z}
$$

where $\alpha \mapsto k_{\alpha}$ is a $W$-invariant function on $R$.

Proof: For all root systems except $A_{1}, B_{2}$, there exists a subsystem isomorphic to $A_{2}$ (see [10]) All other two-dimensional subsystems are isomorphic either to $A_{2}$ or $B_{2}$ (the exceptional case of $G_{2}$ was considered above). Continuing as in the $B_{3}$ case, we complete the proof. 
In the rest of this section we consider the invariant $B_{2}$ case. In other words, we want to find the general solution of the equation (12)

$$
f(x)(g(x+y)+g(x-y))+f(y)(g(x+y)-g(x-y))=0,
$$

which are meromorphic in the vicinity of the origin.

There are the following obvious solutions: $f(z) \equiv 0, g$ an arbitrary function, and $g(z) \equiv 0, f$ an arbitrary function. We call these solutions trivial.

Theorem 6 The general non-trivial solution of the functional equation (13) has the form

$$
g(z)=\frac{A}{\operatorname{sn}(\alpha z, k)} \quad, \quad f(z)=B(\log g(z))^{\prime}
$$

or, more explicitly

$$
g(z)=\frac{A}{\operatorname{sn}(\alpha z, k)} \quad, \quad f(z)=\frac{B}{\operatorname{sn}(\epsilon \alpha z, \tilde{k})},
$$

where $\tilde{k}=(1-k) /(1+k), \epsilon=-i(1+k)$, and $A, B$ and $\alpha$ are arbitrary constants.

Here $\operatorname{sn}(z, k)$ is the classical Jacobi elliptic function (see, e.g., [11]). In the degenerate cases we have the following solutions:

$$
\begin{aligned}
& f(z)=\frac{A}{\sin \alpha z}, \quad g(z)=B \cot \frac{\alpha z}{2}, \\
& f(z)=A \cot \alpha z, \quad g(z)=\frac{B}{\sin \alpha z}, \\
& f(z)=\frac{A}{z}, \quad g(z)=\frac{B}{z},
\end{aligned}
$$

with arbitrary constants $A, B, \alpha$. In particular, we see that all non-trivial solutions are odd functions, and thus lead to invariant Dunkl operators for root systems of type $B_{2}$.

We proceed to prove the Theorem. 
Lemma 7 If $f(z), g(z)$ satisfy the equation (13), then the same is true for $\tilde{f}(z), \tilde{g}(z)$, where

$$
\text { (i) } \quad \tilde{f}(z)=\lambda f(\alpha z), \quad \tilde{g}(z)=\mu g(\alpha z),
$$

for arbitrary constants $\lambda, \mu, \alpha$, or

$$
\tilde{f}(z)=g(z), \quad \tilde{g}(z)=f(z / 2) .
$$

Proof: The first symmetry is evident. To prove the second one, it is sufficient to change variables

$$
u=x+y, \quad v=x-y .
$$

Then equation (13) takes the form

$$
g(u)\left(f\left(\frac{u+v}{2}\right)+f\left(\frac{u-v}{2}\right)\right)+g(v)\left(f\left(\frac{u+v}{2}\right)-f\left(\frac{u-v}{2}\right)\right)=0 .
$$

We will use these symmetries again and again, in particular in the proof of the following

Lemma 8 There are no non-trivial solutions of (13), for which $f(z)$ or $g(z)$ is regular at the origin.

Proof: Because of the symmetry (16), it is enough to consider only the case when $f(z)$ is regular at the origin. Putting $y=0$ in (匹3), we get $2 f(x) g(x)=0$, which means that the solution is trivial.

Lemma 9 Non-trivial solutions of (13) are odd functions:

$$
f(-z)=-f(z), \quad g(-z)=-g(z)
$$

Proof: Rewrite the equation (13) in the form

$$
\phi(y)(g(x+y)+g(x-y))+\phi(x)(g(x+y)-g(x-y))=0,
$$

where $\phi(z)=1 / f(z)$ is regular at vanishes at $z=0$. By putting $x=0$ in this relation, we obtain

$$
\phi(y)(g(y)+g(-y))=0
$$


which implies for a non-trivial solution

$$
g(-y)=-g(y) .
$$

The fact that $f$ is odd follows then from the symmetry (16).

Let us introduce $\lambda=\phi^{\prime}(0)$, where $\phi(z)=1 / f(z)$, as before.

Lemma 10 If $f(z), g(z)$ is a non-trivial solution of (13), then

$$
\begin{aligned}
& \text { (i) } \lambda=\phi^{\prime}(0) \neq 0, \\
& \text { (ii) } \frac{g^{\prime}(x)}{g(x)}=-\lambda f(x), \\
& \text { (iii) } \lambda f(x+y)=\frac{f^{\prime}(y) f(x)-f^{\prime}(x) f(y)}{f^{2}(x)-f^{2}(y)} .
\end{aligned}
$$

Proof: Rewrite equation (13) in the form

$$
g(x+y)=\frac{f(y)-f(x)}{f(y)+f(x)} g(x-y) .
$$

Taking the logarithm of both sides and applying the operator $\partial_{x}+\partial_{y}$, gives

$$
\begin{aligned}
\frac{g^{\prime}(x+y)}{g(x+y)} & =\frac{f(x) f^{\prime}(y)-f(y) f^{\prime}(x)}{f^{2}(y)-f^{2}(x)} \\
& =\frac{\phi^{\prime}(x) \phi(y)-\phi^{\prime}(y) \phi(x)}{\phi^{2}(x)-\phi^{2}(y)} .
\end{aligned}
$$

Putting $y=0$ in (21) implies

$$
\frac{g^{\prime}(x)}{g(x)}=-\lambda f(x) .
$$

In particular, if $\lambda=0, g$ is constant and therefore regular, which is impossible. Now the formula (18) follows from (17) and (20)

Using the symmetry (15), (16), we may set without loss of generality $\lambda=1$, so that

$$
f(z)=\frac{1}{z}+O(z), \quad g(z)=\frac{1}{z}+O(z), \quad(z \rightarrow 0) .
$$


The function $f$ satisfies the functional equation (addition theorem)

$$
f(x+y)=\frac{f(x) f^{\prime}(y)-f(y) f^{\prime}(x)}{f^{2}(x)-f^{2}(y)} .
$$

Rewrite it in the following form

$$
f(x+y)=\frac{\phi^{\prime}(y) f(x)+f^{\prime}(x) \phi(y)}{1-\phi^{2}(y) f^{2}(x)},
$$

and expand the right hand side near $y=0$, using $\phi(z)=z+a z^{3}+O\left(z^{5}\right)$ :

$$
f(x+y)=f(x)+f^{\prime}(x) y+\left(3 a f(x)+f^{3}(x)\right) y^{2}+O\left(y^{3}\right) .
$$

By comparing this with the Taylor expansion, one has

$$
f^{\prime \prime}(x)=2 f^{3}(x)+6 a f(x)
$$

implying, after multiplication by $f^{\prime}$ and integration, that

$$
\left(f^{\prime}\right)^{2}=f^{4}+6 a f^{2}+b
$$

for some constant $b$. The function $\phi=1 / f$ is thus a regular odd solution of the equation

$$
\left(\phi^{\prime}\right)^{2}=1+6 a \phi^{2}+b \phi^{4}
$$

and therefore coincides with the Jacobi elliptic function

$$
\phi(x)=\frac{\operatorname{sn}(\epsilon x, k)}{\epsilon},
$$

where $\left(1+k^{2}\right) \epsilon^{2}=-6 a, k^{2} \epsilon^{4}=b$. Recall that sn is the solution of the equation $\left(s^{\prime}\right)^{2}=\left(1-s^{2}\right)\left(1-k^{2} s^{2}\right)$, with initial condition $s(0)=0$. It satisfy the addition formula discovered by A. Cayley (see [1] )

$$
s(x+y)=\frac{s^{2}(x)-s^{2}(y)}{s(x) s^{\prime}(y)-s(y) s^{\prime}(x)}
$$

This implies the relation (22) for $f(x)=\epsilon / \operatorname{sn}(\epsilon x, k)$. Note that by (16), also $g$ has the same form as $f$, in general with different values of $\epsilon$ and $k$. 
Lemma 11 If $f(x)=\epsilon / \operatorname{sn}(\epsilon x, k)$ and $g^{\prime}(x) / g(x)=-f(x)$ then $f(x), g(x)$ are a non-trivial solution of the functional equation (13).

Proof: We have

$$
\begin{aligned}
2 \frac{g^{\prime}(x+y)}{g(x+y)} & =-2 f(x+y) \\
& =2 \frac{f^{\prime}(x) f(y)-f^{\prime}(y) f(x)}{f^{2}(x)-f^{2}(y)} \\
& =\frac{f^{\prime}(y)-f^{\prime}(x)}{f(y)-f(x)}-\frac{f^{\prime}(y)+f^{\prime}(x)}{f(y)+f(x)}
\end{aligned}
$$

So

$$
\left(\partial_{x}+\partial_{y}\right) \log g(x+y)=\left(\partial_{x}+\partial_{y}\right) \log \frac{f(y)-f(x)}{f(y)+f(x)} .
$$

This means that

$$
g(x+y)=\frac{f(y)-f(x)}{f(y)+f(x)} \psi(x-y)
$$

for some function $\psi$. Rewriting this equation as

$$
g(x+y)=\frac{\phi(x)-\phi(y)}{\phi(x)+\phi(y)} \psi(x-y), \quad \phi=1 / f,
$$

and putting $y=0$, gives $\psi(x)=g(x)$, and therefore

$$
g(x+y)=\frac{f(y)-f(x)}{f(y)+f(x)} g(x-y),
$$

which is equivalent to (13).

To finish the proof of Theorem 6, we have to prove that the relation between $k, \tilde{k}, \epsilon$ in

$$
g(x)=\frac{1}{\operatorname{sn}(x, k)}, \quad f(x)=\frac{\epsilon}{\operatorname{sn}(\epsilon x, \tilde{k})},
$$

where $g^{\prime} / g=-f$ is the one given in Theorem 6 .

The function $1 / f=-g / g^{\prime}=\operatorname{sn}(\epsilon x, \tilde{k}) / \epsilon$ satisfies the equation

$$
\left[\left(g / g^{\prime}\right)^{\prime}\right]^{2}=\left[1-\epsilon^{2}\left(g / g^{\prime}\right)^{2}\right]\left[1-\tilde{k}^{2} \epsilon^{2}\left(g / g^{\prime}\right)^{2}\right]
$$


or, equivalently,

$$
\left(\left(g^{\prime}\right)^{2}-g g^{\prime \prime}\right)^{2}=\left(\left(g^{\prime}\right)^{2}-\epsilon^{2} g^{2}\right)\left(\left(g^{\prime}\right)^{2}-\tilde{k}^{2} \epsilon^{2} g^{2}\right) .
$$

Substituting $\left(g^{\prime}\right)^{2}=\left(g^{2}-1\right)\left(g^{2}-k^{2}\right), g^{\prime \prime}=2 g^{3}-\left(k^{2}+1\right) g$, into (28), gives the following two possibilities

(i) $2 k=-\left(k^{2}+1\right)-\epsilon^{2},-2 k=-\left(k^{2}+1\right)-\tilde{k}^{2} \epsilon^{2}$

(ii) $-2 k=-\left(k^{2}+1\right)-\epsilon^{2}, 2 k=-\left(k^{2}+1\right)-\tilde{k}^{2} \epsilon^{2}$

In the first case, we get $\tilde{k}=(1-k) /(1+k), \epsilon=i(k+1)$, as required. The second case leads to an equivalent answer. Theorem 6 is proved.

Remark. The transformation of elliptic functions $g \rightarrow f$ is of second order and therefore can be reduced to the well-known Landen transformation (see [11]). One can check that it is the composition of the "imaginary Jacobi transformation", which is the unimodular transformation with the action on the homology of the curve described by the matrix $J$

$$
J=\left(\begin{array}{cc}
0 & 1 \\
-1 & 0
\end{array}\right)
$$

and Landen's transformation with matrix $L$,

$$
L=\left(\begin{array}{ll}
2 & 0 \\
0 & 1
\end{array}\right)
$$

Let us call it LJ-transformation. Thus the functional equation (13) describes a pair of elliptic functions related by the LJ-transformation. This fact has found recently an interesting topological application in the theory of elliptic genera [4].

Note that $f$ and $g$, as elliptic functions of second order "live" on different elliptic curves, one of which is a double cover of the other. In particular, the solutions found here are not special cases of the elliptic solutions considered in the next Section. 


\section{Elliptic Dunkl operators}

In this section we consider only the case where $R$ is the root system of a semisimple Lie algebra with Weyl group $G=W$. Let us consider the elliptic curve with modular parameter $\tau, \operatorname{Im}(\tau)>0$, and the family of functions

$$
\sigma_{\lambda}(z)=\frac{\theta_{1}(z-\lambda) \theta_{1}^{\prime}(0)}{\theta_{1}(z) \theta_{1}(-\lambda)}, \quad \lambda \in \mathbf{C} \backslash \mathbf{Z}+\tau \mathbf{Z},
$$

given in terms of Jacobi's theta function

$$
\theta_{1}(z)=-\sum_{n=-\infty}^{\infty} e^{2 \pi i\left(z+\frac{1}{2}\right)\left(n+\frac{1}{2}\right)+\pi i \tau\left(n+\frac{1}{2}\right)^{2}}
$$

The functions $\sigma_{\lambda}$ have the following defining properties:

(i) $\sigma_{\lambda}(z+1)=\sigma_{\lambda}(z)$.

(ii) $\sigma_{\lambda}(z+\tau)=e^{2 \pi i \lambda} \sigma_{\lambda}(z)$.

(iii) $\sigma_{\lambda}$ is meromorphic, its poles are on the lattice $\mathbf{Z}+\tau \mathbf{Z}$, and $\sigma_{\lambda}(z)=$ $1 / z+O(1)$ as $z \rightarrow 0$.

More properties of this functions are given in the Appendix.

Theorem 12 For any generic $\lambda \in V_{\mathbf{C}}=V \otimes_{\mathbf{R}} \mathbf{C}$, and $W$-invariant function $k_{\alpha}$, the functions

$$
f_{\alpha}(z)=k_{\alpha} \sigma_{\left(\alpha^{\vee}, \lambda\right)}(z),
$$

where $\alpha^{\vee}=2 \alpha /(\alpha, \alpha)$, satisfy the functional equations (9).

Proof: Fix the rotation $r$. All roots involved in the left hand side of the functional equation (9)

$$
I(x)=\sum_{\alpha, \beta \in R_{+}: s_{\alpha} s_{\beta}=r}\langle\alpha, \beta\rangle \sigma_{\left(\alpha^{\vee}, \lambda\right)}((\alpha, x)) \sigma_{\left(\beta^{\vee}, \lambda\right)}\left(\left(s_{\alpha}(\beta), x\right)\right)
$$

lie on the same two-dimensional plane. Consider $I(x)$ as a meromorphic function of $x \in V_{\mathbf{C}}$, and let $P^{\vee}=\{p \in V \mid(p, \alpha) \in \mathbf{Z} \forall \alpha \in R\}$. Then if $p \in P^{\vee}, I(x+p)=I(x)$, and as $x \rightarrow x+p \tau$, the term labeled by $(\alpha, \beta)$ in the sum (30) gets multiplied by

$$
e^{2 \pi i\left(\left(\alpha^{\vee}, \lambda\right)(\alpha, p)+\left(\beta^{\vee}, \lambda\right)\left(s_{\alpha}(\beta), p\right)\right)} .
$$


Since $s_{\alpha}(\lambda)=\lambda-\left(\alpha^{\vee}, \lambda\right) \alpha$, we see that the multiplier can be rewritten as

$$
e^{2 \pi i\left(\lambda-s_{\alpha} s_{\beta}(\lambda), p\right)}
$$

and is therefore the same for all terms in the sum. It follows that $I(x)$ has the quasi-periodicity property

$$
I(x+q+p \tau)=e^{2 \pi i(\lambda-r(\lambda), p)} I(x), \quad q+p \tau \in P^{\vee}+\tau P^{\vee} .
$$

Let us now consider the poles of the function $I$. Poles may appear when $x$ is on the hyperplanes $(\alpha, x)=0, \alpha \in R_{+}$, or their translates by $P^{\vee}+\tau P^{\vee}$. If $x$ approaches the hyperplane $(\alpha, x)=0$, the singular terms in the sum (30) are the terms indexed by $\alpha, \beta$ and $\gamma, \delta$ where $s_{\gamma}(\delta)= \pm \alpha$. As all roots are on a plane this implies that $\gamma=\mp \beta$. The sign is $+\left(\right.$ thus $\left.s_{\gamma}(\delta)=-\alpha\right)$ since $\gamma>0$. In particular only two terms are singular in (30). The coefficient of the (simple) pole is (cf. (iii) above)

$$
\langle\alpha, \beta\rangle \sigma_{\left(\beta^{\vee}, \lambda\right)}\left(\left(s_{\alpha}(\beta), x\right)\right)-\langle\beta, \delta\rangle \sigma_{\left(\beta^{\vee}, \lambda\right)}((\beta, x)) .
$$

This expression vanishes on the hyperplane $(\alpha, x)=0$ because $s_{\alpha}(x)=x$ there, and

$$
\langle\beta, \delta\rangle=-\left\langle s_{\beta}(\beta), s_{\beta}(\delta)\right\rangle=-\langle\beta, \alpha\rangle=\langle\alpha, \beta\rangle .
$$

It follows that the singularity at $(\alpha, x)=0$ (and thus on all affine hyperplanes $(\alpha, x)=n+m \tau, n, m \in \mathbf{Z}$ by (31)) is removable. We conclude that $I$ has no singularity on $V_{\mathbf{C}}$, and has the quasi-periodicity property (31). It therefore vanishes, by Fourier series theory.

Corollary 13 The operators

$$
\nabla_{\xi}^{\lambda}=\partial_{\xi}+\sum_{\alpha \in R_{+}} k_{\alpha}(\alpha, \xi) \sigma_{\left(\alpha^{\vee}, \lambda\right)}((\alpha, x)) \hat{s}_{\alpha}
$$

form a commutative family:

$$
\left[\nabla_{\xi}^{\lambda}, \nabla_{\eta}^{\lambda}\right]=0
$$

Let us call these operators elliptic Dunkl operators. They are not $W$-invariant but $W$-equivariant

$$
\hat{w} \nabla_{\xi}^{\lambda} \hat{w}^{-1}=\nabla_{w(\xi)}^{w(\lambda)}
$$


Example: In the $A_{n-1}$ case, we identify functions on $V=\left\{\mathbf{R}^{n} \mid \Sigma_{i} x_{i}=0\right\}$ with functions $f$ on $\mathbf{R}^{n}$ such that $f\left(x_{1}+a, \ldots, x_{n}+a\right)$ is independent of $a \in \mathbf{R}$. Let $e_{1}, \ldots, e_{n}$ be the standard basis of $\mathbf{R}^{n}$ and put

$$
\bar{e}_{i}=e_{i}-\frac{1}{n} \sum_{j=1}^{n} e_{j} \in V .
$$

Then Dunkl operators are linear combinations of the commuting operators $\nabla_{i}^{\lambda}=\nabla_{\bar{e}_{i}}^{\lambda}$ :

$$
\nabla_{i}^{\lambda}=\frac{\partial}{\partial x_{i}}+k \sum_{j: j \neq i} \sigma_{\lambda_{i}-\lambda_{j}}\left(x_{i}-x_{j}\right) \hat{s}_{i j},
$$

where $\hat{s}_{i j}$ is the operator that interchanges the $i$ th and $j$ th variable.

Remark. It is interesting to note that the usual Dunkl operator reminds Moser's L-matrix for the Calogero problem [12], whereas the elliptic Dunkl operator (33) reminds Krichever's generalization [13], see also [14]. The general solution of the corresponding functional equation (different from ours) was found by Bruschi and Calogero [15]. More general functional equations motivated by these problems, as well as some topological problems, were introduced and solved by one of the authors [5], [6].

\section{General solution of the functional equation in the $A_{n-1}$ case}

In this section we show that elliptic Dunkl operators are essentially the only solutions of the functional equation (9), in the $A_{n-1}$ case, $n-1 \geq 2$.

As before, we start with $A_{2}$. In this case, the functional equation is (11)

$$
f(u) g(u+v)+g(v) h(-u)+h(-u-v) f(-v)=0 .
$$

We must find the most general solutions of this functional equation, where $f$, $g$, and $h$ are assumed to be meromorphic functions defined in a neighborhood of the origin.

First of all, if one of the three functions vanishes identically, then the functional equation says that the product of the other two vanishes, and we get a solution where two functions vanish and the third is arbitrary. We call these solution trivial, and consider from now on only solutions where $f, g$ and $h$ are not identically zero. 
Lemma 14 If $f, g$, h satisfy (34), then (i) $\tilde{f}(z)=g(z), \tilde{g}(z)=h(z), \tilde{h}(z)=$ $f(z)$, (ii) $\tilde{f}(z)=a f(b z) e^{\alpha z}, \tilde{g}(z)=a g(b z) e^{\beta z}, \tilde{h}(z)=a f(b z) e^{\gamma z}$, for arbitrary constants $a, \ldots, \gamma$ such that $\alpha+\beta+\gamma=0$,

also satisfy (34).

Proof: Replacing $(u, v)$ by $(-u-v, u)$ in (34) implies (i). Property (ii) is easy to check.

Proposition 15 The only non-trivial solutions of (34) holomorphic around the origin are

$$
f(u)=a e^{\alpha u}, \quad g(u)=b e^{\beta u}, \quad h(u)=c e^{\gamma u},
$$

where $a, b, c \neq 0, a b+b c+a c=0, \alpha+\beta+\gamma=0$.

Proof: It is easy to check that these are solutions. We prove uniqueness. Suppose $f, g, h$ are a non-trivial solution of (34), defined and holomorphic in a neighborhood of the origin. Taking $v=0$ in (34), we get

$$
f(u) g(u)+(f(0)+g(0)) h(-u)=0 .
$$

We have $f(0)+g(0) \neq 0$ since $f g$ does not vanish identically. Similarly, by Lemma 14,

$$
\begin{aligned}
g(u) h(u)+(g(0)+h(0)) f(-u) & =0 \\
h(u) f(u)+(h(0)+f(0)) g(-u) & =0 .
\end{aligned}
$$

Introduce the functions $F(u)=f(u) f(-u), G(u)=g(u) g(-u), H(u)=$ $h(u) h(-u)$, and $S(u)=f(u) g(u) h(u)$. Multiplying (35), (36), (37) by $h(u)$, $f(u)$ and $g(u)$, respectively, we obtain

$$
S(u)=\lambda F(u)=\mu G(u)=\nu H(u),
$$

for some non-zero constants $\lambda, \mu, \nu$. In particular $S$ is an even function, and thus

$$
S(u)^{2}=S(u) S(-u)=F(u) G(u) H(u) .
$$

Hence $F(u)^{3}=\operatorname{const} F(u)$, and $F$ is a constant. Similarly, $G$ and $H$ are constant functions. Thus we have

$$
f(u) f(-u)=a^{2}, \quad g(u) g(-u)=b^{2}, \quad h(u) h(-u)=c^{2},
$$


for some constants $a, b, c \neq 0$. We can therefore write

$$
f(u)=a e^{\phi(u)}, \quad g(u)=b e^{\psi(u)}, \quad h(u)=c e^{\eta(u)},
$$

for some odd functions $\phi, \psi, \eta$, holomorphic in a neighborhood of 0 . Since $S(u)=f(u) g(u) h(u)$ is constant, we have $\phi+\psi+\eta=0$. Inserting (39) in the functional equation gives

$$
a b e^{\phi(u)+\psi(u+v)}+b c e^{\psi(v)-\eta(u)}+a c e^{-\eta(u+v)-\phi(v)}=0,
$$

which, after elimination of $\eta=-\phi-\psi$ can be recast in the more convenient form

$$
a b+b c \Psi(u, v)+a c \Phi(u, v)^{-1}=0,
$$

with $\Phi(u, v)=\exp (\phi(u+v)-\phi(u)-\phi(v))$, and $\Psi(u, v)=\exp (\psi(u+v)-\psi(u)-$ $\psi(v))$. In particular, setting $u=v=0$, we obtain the condition $a b+b c+a c=$ 0 . Moreover, we have $\Phi(-u,-v)=\Phi(u, v)^{-1}$, and $\Psi(-u,-v)=\Psi(u, v)^{-1}$, since $\phi$ and $\psi$ are odd functions. Replacing $(u, v)$ by $(-u,-v)$ in (40) yields the equation

$$
a b+b c \Psi(u, v)^{-1}+a c \Phi(u, v)=0 .
$$

Elimination of $\Psi$ from (40), (41) gives a non trivial quadratic equation with constant coefficients for $\Phi$. Thus $\Phi$ is constant, implying that $\Psi$ is constant as well. we conclude that $\phi(u+v)=\phi(u)+\phi(v)$, and $\psi(u+v)=\psi(u)+\psi(v)$, which leaves us with the solution $\phi(u)=\alpha u, \psi(u)=\beta u$.

From now on, we consider the case when $f(u)$ has a pole of order $p>0$ at the origin. It is easy to see that $h$ and $g$ also have a pole of the same order $p$ at $u=0$.

Inserting the expansion

$$
\begin{aligned}
f(u) & =\frac{a}{u^{p}}+\cdots, \\
g(u) & =\frac{b}{u^{p}}+\cdots, \\
h(u) & =\frac{c}{u^{p}}+\cdots,
\end{aligned}
$$

into (34), and multiplying the relation by $u^{p}(u+v)^{p} v^{p}$, yields a series in $u$ and $v$ which starts as

$$
a c u^{p}+a b v^{p}+(-1)^{p} b c(u+v)^{p} .
$$


We see that $p=1$ is the only case in which cancellation is possible. In this case,

$$
a c-b c=0, \quad a b-b c=0,
$$

which implies $a=b=c$. Without loss of generality, we consider the case $a=b=c=1$.

Suppose that $f, g, h$ are a solution of (34) with a simple pole at the origin with unit residue:

$$
\begin{aligned}
f(u) & =\frac{1}{u}+f_{0}+\cdots, \\
g(u) & =\frac{1}{u}+g_{0}+\cdots, \\
h(u) & =\frac{1}{u}+h_{0}+\cdots
\end{aligned}
$$

The left hand side of (34) has a Laurent expansion at $v=0$ :

$$
f(u) g(u)+\left(\frac{1}{v}+g_{0}\right) h(-u)+\left(h(-u)-v h^{\prime}(-u)\right)\left(-\frac{1}{v}+f_{0}\right)+O(v) .
$$

The constant term gives the equation

$$
f(u) g(u)+\left(f_{0}+g_{0}\right) h(-u)+h^{\prime}(-u)=0 .
$$

Similarly, by Lemma 14 (i),

$$
\begin{aligned}
g(u) h(u)+\left(g_{0}+h_{0}\right) f(-u)+f^{\prime}(-u) & =0, \\
h(u) f(u)+\left(h_{0}+f_{0}\right) g(-u)+g^{\prime}(-u) & =0 .
\end{aligned}
$$

Introduce $F(u)=f(u) f(-u), G(u)=g(u) g(-u), H(u)=h(u) h(-u)$, and $S(u)=f(u) g(u) h(u)$, as above. Note that $F, G$ and $H$ are even functions. Then $F^{\prime}(u)=f^{\prime}(u) f(-u)-f^{\prime}(-u) f(u)$, and (49) implies that $F^{\prime}(u)=$ $S(u)-S(-u)$. More generally, (48 50) imply

$$
F^{\prime}(u)=G^{\prime}(u)=H^{\prime}(u)=S(u)-S(-u)
$$

We can then write

$$
F(u)=a-P(u), \quad G(u)=b-P(u), \quad H(u)=c-P(u),
$$


where $P(u)=1 / u^{2}+O\left(u^{2}\right)$ has no constant term, and $a, b, c$ are integration constants. The even function $P$ obeys

$$
P^{\prime}(u)=-S(u)+S(-u) .
$$

Let us compute the derivative $S^{\prime}=\left(f^{\prime} / f+g^{\prime} / g+h^{\prime} / h\right) S$ using (48 50)

$$
\begin{aligned}
S^{\prime}(u) & =\alpha S(u)-G(u) H(u)-H(u) F(u)-F(u) G(u) \\
& =\alpha S(u)-3 P(u)^{2}+\beta P(u)+\gamma
\end{aligned}
$$

for some constants $\alpha, \beta, \gamma$. Let us consider the even and odd part of this equation separately:

$$
\begin{aligned}
\frac{d}{d u}(S(u)+S(-u)) & =\alpha(S(u)-S(-u))=-\alpha P^{\prime}(u) \\
\frac{d}{d u}(S(u)-S(-u)) & =\alpha(S(u)+S(-u))-6 P(u)^{2}+2 \beta P(u)+2 \gamma
\end{aligned}
$$

Integrating (53) gives

$$
S(u)+S(-u)=-\alpha P(u)+\delta,
$$

for some $\delta$. By subtracting (52) from this equation, we obtain

$$
S(u)=\frac{1}{2}\left(-\alpha P(u)+\delta-P^{\prime}(u)\right) .
$$

Inserting (55) and (52) into (54) yields finally the differential equation

$$
P^{\prime \prime}(u)=6 P(u)^{2}+\left(\alpha^{2}-2 \beta\right) P(u)-\alpha \delta-2 \gamma .
$$

Let us multiply this equation by $P^{\prime}(u)$ and integrate. We get

$$
P^{\prime}(u)^{2}=4 P(u)^{3}+c_{1} P(u)^{2}+c_{2} P(u)+c_{3} .
$$

In fact $c_{1}=0$, since, by construction, the Laurent expansion of $P$ has no constant term. This equation is well-known to have a unique meromorphic solution with double pole at the origin. It is the Weierstrass function $\wp$ for some elliptic curve determined by the coefficients $c_{2}, c_{3}$, or one of its degenerations $\omega^{2}\left(1 / \sin (\omega u)^{2}-1 / 3\right), 1 / u^{2}$ (see [1]]). After rescaling of the 
variables as in Lemma 14 if necessary, we may assume that the periods of $\wp$ are 1 and $\tau$, and that $\omega=\pi$. By writing the constants $a, b, c$ in (51) as $P(\lambda), P(\mu), P(\nu)$ respectively (this is always possible since $P$ defines a surjective map from the elliptic curve $\mathbf{C} / \mathbf{Z}+\tau \mathbf{Z}$, or, in the degenerate cases, $(\mathbf{C} / \mathbf{Z}) \cup\{i \infty\}, \mathbf{C} \cup\{i \infty\}$, onto the Riemann sphere), we see that the equation $f(u) f(-u)=P(\lambda)-P(u)$ has the solution (see the Appendix)

$$
f(u)=\sigma_{\lambda}(u)
$$

and, in the degenerate cases,

$$
\begin{aligned}
f(u) & =\frac{\pi \sin (\pi(u-\lambda))}{\sin (\pi u) \sin (-\pi \lambda)}=\pi(\cot (\pi u)-\cot (\pi \lambda)), \\
f(u) & =\frac{1}{u}-\frac{1}{\lambda} .
\end{aligned}
$$

The general solution in a neighborhood of the origin is $f(u) \exp \phi(u)$ for some odd function $\phi(u)$ regular at the origin. Similar formulas hold for $g$ and $h$ : the functions

$$
f(u)=\sigma_{\lambda}(u), \quad g(u)=\sigma_{\mu}(u), \quad h(u)=\sigma_{\nu}(u),
$$

and their degenerations, are a solution of the functional equation, provided that $\lambda+\mu+\nu=0$ (modulo the lattice). We now show that these are the only (up to transformations of Lemma 14 (ii)) solutions such that (51) holds with $a=P(\lambda), b=P(\mu), c=P(\nu)$. Suppose $\tilde{f}, \tilde{g}, \tilde{h}$ is another solution. Then

$$
\tilde{f}(u)=e^{\phi(u)} f(u), \quad \tilde{g}(u)=e^{\psi(u)} g(u), \quad \tilde{h}(u)=e^{\eta(u)} h(u),
$$

for some odd functions $\phi, \psi, \eta$. Since the product $S=f g h$ is expressed in terms of $P$ (see (56)), the sum $\phi+\psi+\eta$ vanishes identically. Inserting (57) in (49), (50), we deduce immediately that $\phi^{\prime}(u)=\psi^{\prime}(u)=0$ for all $u$. Thus $\phi$ and $\psi$ are linear functions.

Combining this result with Prop. 15, we obtain the following theorem.

Theorem 16 The following list exhausts all non-trivial solutions of the functional equation

$$
f(u) g(u+v)+g(v) h(-u)+h(-u-v) f(-v)=0 .
$$


Elliptic solutions:

$$
f(u)=a \sigma_{\lambda}(b u) e^{\alpha u}, \quad g(u)=a \sigma_{\mu}(b u) e^{\beta u}, \quad h(u)=a \sigma_{\nu}(b u) e^{\gamma u},
$$

where the function $\sigma_{\lambda}$ is defined in (29).

Trigonometric solutions:

$$
\begin{gathered}
f(u)=\frac{a \sin (b(u-\lambda))}{\sin (b u) \sin (-b \lambda)} e^{\alpha u}, \quad g(u)=\frac{a \sin (b(u-\mu))}{\sin (b u) \sin (-b \mu)} e^{\beta u}, \\
h(u)=\frac{a \sin (b(u-\nu))}{\sin (b u) \sin (-b \nu)} e^{\gamma u}
\end{gathered}
$$

Rational solutions:

$$
f(u)=\left(\frac{a}{u}-\frac{1}{\lambda}\right) e^{\alpha u}, \quad g(u)=\left(\frac{a}{u}-\frac{1}{\mu}\right) e^{\beta u}, \quad h(u)=\left(\frac{a}{u}-\frac{1}{\nu}\right) e^{\gamma u} .
$$

Regular solutions:

$$
f(u)=-\frac{1}{\lambda} e^{\alpha u}, \quad g(u)=-\frac{1}{\mu} e^{\beta u}, \quad h(u)=-\frac{1}{\nu} e^{\gamma u} .
$$

The parameters in these solutions are complex numbers $a, b, \alpha, \beta, \gamma, \lambda, \mu$, $\nu$ satisfying $\alpha+\beta+\gamma=0, \lambda+\mu+\nu=0$.

In the trigonometric and rational cases, the limiting cases where $\lambda, \mu, \nu$ take the value $\pm i \infty$ are permitted.

Remark. Notice that all these solutions are limiting cases (degenerations) of the elliptic solutions.

This theorem covers the case $A_{2}$, but gives immediately the answer in the case $A_{n-1}, n-1 \geq 2$. In this case, we identify, as above, functions on $V$ with translation invariant functions on $\mathbf{R}^{n}$.

Corollary 17 Let $n \geq 3$. The $n$ operators

$$
\nabla_{i}=\partial_{i}+\sum_{j: j \neq i} f_{i j}\left(x_{i}-x_{j}\right) \hat{s}_{i j}, \quad i=1, \ldots, n,
$$

with $f_{i j} \not \equiv 0, f_{i j}(u)=-f_{j i}(-u)$ are pairwise commutative iff $f_{i j}(x)=$ $k \sigma_{\lambda_{i}-\lambda_{j}}(b x) e^{\left(\alpha_{i}-\alpha_{j}\right) x}$ where $\sigma_{\lambda}$ is the function defined in (29), or one of its degenerations (see above). 


\section{Quantum Dunkl operators}

Fix complex parameters $\tau, \mu$ and $\kappa$ such that $\operatorname{Im}(\tau)>0, \mu \notin \mathbf{Z}+\tau \mathbf{Z}$ and $\kappa \neq 0$. Consider the operator $R(\lambda)$, depending on the "spectral parameter" $\lambda \in \mathbf{C}$, and acting on the space of, say, meromorphic functions of two complex variables $x_{1}$ and $x_{2}$ :

$R(\lambda) f\left(x_{1}, x_{2}\right)=\frac{1}{\sigma_{\mu}(\lambda)}\left\{\sigma_{\mu}\left(x_{12}+\frac{\mu}{\kappa}\right) f\left(x_{1}+\frac{\mu}{\kappa}, x_{2}-\frac{\mu}{\kappa}\right)-\sigma_{\lambda}\left(x_{12}+\frac{\mu}{\kappa}\right) f\left(x_{2}, x_{1}\right)\right\}$.

We use the notation $x_{12}$ to denote the difference $x_{1}-x_{2}$. The operator $R$ obeys the quantum Yang-Baxter equation

$$
R\left(\lambda_{12}\right)^{(12)} R\left(\lambda_{13}\right)^{(13)} R\left(\lambda_{23}\right)^{(23)}=R\left(\lambda_{23}\right)^{(23)} R\left(\lambda_{13}\right)^{(13)} R\left(\lambda_{12}\right)^{(12)} .
$$

The two sides of this equation are operators acting on functions of three variables, and the notation $R(\lambda)^{(i j)}$ indicates the operator $R(\lambda)$ acting on a function of several variables, by viewing it as a function of the $i$ th and $j$ th variable. This solution of the Yang-Baxter equation is essentially the one introduced in [8] as three-parameter generalization of the two-parameter solution of Shibukawa and Ueno [7]. For positive integer values of $\kappa$ it admits a restriction to a finite dimensional subspace coinciding with Belavin's solution [16].

With the normalization used here we have "unitarity"

$$
R\left(\lambda_{12}\right)^{(12)} R\left(\lambda_{21}\right)^{(21)}=\mathrm{Id},
$$

and $R(0) f\left(x_{1}, x_{2}\right)=f\left(x_{2}, x_{1}\right)$. Moreover $R(\lambda)$ tends to the identity as $\mu$ goes to zero.

Solutions of the quantum Yang-Baxter equation with these properties can be used to construct commuting operators $T_{i}\left(\lambda_{1}, \ldots, \lambda_{n}\right)$ (related to transfer matrices of integrable models of statistical mechanics):

$$
T_{i}\left(\lambda_{1}, \ldots, \lambda_{n}\right)=R^{(i, i+1)} \cdots R^{(i, n)} R^{(i, 0)} \cdots R^{(i, i-1)} .
$$

These operators act on functions of $n$ variables, and, as before, $R^{(i j)}=R\left(\lambda_{i}-\right.$ $\left.\lambda_{j}\right)^{(i j)}$ is the operator $R\left(\lambda_{i}-\lambda_{j}\right)$ acting on a function of $n$ variables by viewing it as a function of the $i$ th and $j$ th variable.

Our result is that elliptic Dunkl operator $\nabla_{i}^{\lambda}$ defined in (33) can be obtained as semiclassical limit of the quantum operators, if $k$ is integer. 
Theorem 18 Let $T_{i}\left(\lambda_{1}, \ldots, \lambda_{n}\right)$ be the operators (61) acting on functions $f$ on $\mathbf{R}^{n}$, such that $f\left(x_{1}+a, \ldots, x_{n}+a\right)=f\left(x_{1}, \ldots, x_{n}\right)$ for all $a \in \mathbf{R}$. For all integer $k$, we have

$$
T_{i}\left(\lambda_{1}, \ldots, \lambda_{n}\right)=\mathrm{Id}+\frac{n}{k} \mu g_{\kappa / n}^{-1} \nabla_{i}^{\lambda} g_{\kappa / n}+O\left(\mu^{2}\right),
$$

where $g_{m}$ is the function

$$
g_{m}(x)=\prod_{i<j} \theta_{1}\left(x_{i j}\right)^{m} \exp \left(m \sum_{i \neq j} x_{i} \theta_{1}^{\prime}\left(\lambda_{i j}\right) / \theta_{1}\left(\lambda_{i j}\right)\right)
$$

viewed as multiplication operator, and the parameter $\kappa$ of $R$ is given by $\kappa=$ $(-1)^{k} n k$.

Proof: Let $\rho(x)=\theta_{1}^{\prime}(x) / \theta_{1}(x)$. We first compute the expansion of $R$ to first order.

$$
R(\lambda)=\mathrm{Id}+\mu r(\lambda)+O\left(\mu^{2}\right),
$$

where $r$, the "classical $r$-matrix", is the differential-difference operator

$$
r(\lambda)=\frac{1}{\kappa}\left(\partial_{1}-\partial_{2}\right)+\sigma_{\lambda}\left(x_{12}\right) \hat{s}_{12}+\rho(\lambda)-\rho\left(x_{12}\right) .
$$

This implies that

$$
T_{i}\left(\lambda_{1}, \ldots, \lambda_{n}\right)=\mathrm{Id}+\mu \sum_{j: j \neq i} r^{(i j)}\left(\lambda_{i j}\right)+O\left(\mu^{2}\right) .
$$

Since $\Sigma_{1}^{n} \partial_{i} f=0$, we have

$$
\sum_{j: j \neq i}\left(\partial_{i}-\partial_{j}\right) f=n \partial_{i} f
$$

and therefore

$T_{i}\left(\lambda_{1}, \ldots, \lambda_{n}\right)=\mathrm{Id}+\frac{n}{\kappa} \mu\left(\partial_{i}+\sum_{j: j \neq i} \frac{\kappa}{n} \sigma_{\lambda_{i j}}\left(x_{i j}\right) \hat{s}_{i j}+\sum_{j: j \neq i} \frac{\kappa}{n}\left(\rho\left(\lambda_{i j}\right)-\rho\left(x_{i j}\right)\right)\right)$.

The claim follows then from the relations

$$
\begin{aligned}
\partial_{i} g_{m}(x) & =m \sum_{j: j \neq i}\left(\rho\left(\lambda_{i j}\right)-\rho\left(x_{i j}\right)\right), \\
\hat{s}_{i j} g_{m} & =(-1)^{m} g_{m} \hat{s}_{i j},
\end{aligned}
$$

and the elementary fact that $m=(-1)^{k} k$ obeys $(-1)^{m} m=k$. 


\section{Quantum n-body problems}

Trigonometric and rational Dunkl operators in the $A_{n-1}$ are used in the theory of integrable quantum $n$-body problems of the Calogero-Sutherland type, see [9], [3]. Let us consider the trigonometric Dunkl operators (in a slightly different normalization)

$$
\nabla_{i}=\partial_{i}+\sum_{j: j \neq i} k\left(\operatorname{coth}\left(x_{i}-x_{j}\right)-1\right) \hat{s}_{i j}
$$

Then the operators $L_{j}=\operatorname{Res} \sum_{i=1}^{n}\left(\nabla_{i}\right)^{j}, j=1, \ldots, n$ form a set of pairwise commuting $S_{n}$-invariant differential operators, and generate the algebra of $S_{n}$-invariant differential operators commuting with the Schrödinger operator

$$
L_{2}=\sum_{i=1}^{n} \partial_{i}^{2}-k(k+1) \sum_{i \neq j} \frac{1}{\sinh ^{2}\left(x_{i}-x_{j}\right)} .
$$

Here Res $M$ is the differential operator whose restriction to $S_{n}$-invariant functions coincides with the differential-difference operator $M$.

In the elliptic case, we are lead to consider the commuting operators $M_{j}(\lambda)=\sum_{i=1}^{n}\left(\nabla_{i}^{\lambda}\right)^{j}$, where $\nabla_{i}^{\lambda}$ is the elliptic Dunkl operator (33). These operators are not $S_{n}$-invariant. Instead, they obey $\hat{w} M_{j}(\lambda) \hat{w}^{-1}=M_{j}(w(\lambda))$. However, let us consider the singular limit when $\lambda$ tends to the symmetric point 0 , for $j=2$. We use the shorthand notation $x_{i j}=x_{i}-x_{j}$.

$$
\begin{aligned}
M_{2}(\lambda) & =\sum_{i=1}^{n}\left(\nabla_{i}^{\lambda}\right)^{2} \\
& =\sum_{i=1}^{n} \partial_{i}^{2}+k \sum_{i \neq j} \sigma_{\lambda_{i j}}^{\prime}\left(x_{i j}\right) \hat{s}_{i j}+k^{2} \sum_{i \neq j} \sigma_{\lambda_{i j}}\left(x_{i j}\right) \sigma_{\lambda_{i j}}\left(x_{j i}\right) .
\end{aligned}
$$

In this calculation, we used the functional equation of Prop. 19 (iii) for $\sigma_{\lambda}$ and the fact that $\sigma_{-\lambda}(-x)=-\sigma_{\lambda}(x)$. Since

$$
\sigma_{\lambda}(x) \sigma_{\lambda}(-x)=\wp(\lambda)-\wp(x)
$$

and $\lim _{\lambda \rightarrow 0} \sigma_{\lambda}^{\prime}(x)=-\wp(x)-2 \eta_{1}$, (see the Appendix) we see that

$$
M_{2}(\lambda)-\sum_{i \neq j} \wp\left(\lambda_{i j}\right)
$$


has a limit as $\lambda \rightarrow 0$ :

$$
L_{2}=\lim _{\lambda \rightarrow 0}\left(M_{2}(\lambda)-\sum_{i \neq j} \wp\left(\lambda_{i j}\right)=\sum_{i=1}^{n} \partial_{i}^{2}-k(k+1) \sum_{i \neq j} \wp\left(x_{i}-x_{j}\right)+\right.\text { const. }
$$

This is (minus) the Schrödinger operator of the so-called elliptic CalogeroMoser integrable $n$-body problem (see, e.g., [21]).

It is reasonable to conjecture that the higher $S_{n}$-invariant differential operators commuting with $L_{2}$ can be obtained as $\lambda \rightarrow 0$ limits of suitable equivariant polynomials in $\nabla_{i}^{\lambda}$ with $\lambda$ dependent coefficients.

For integer $k$ there is a conjecture of one of the authors (see [17]) that there are additional integrals of motion, such that the whole ring of quantum integral is supercomplete. We hope that elliptic Dunkl operators will help to prove it.

Another interesting problem is to understand the analogue of Opdam's shift operator in the elliptic case (cf. [18]). The one-dimensional case shows that it can not be a pure differential operator, because the genera of the spectral curves for Lamé operators depend on the integer parameter $k$.

In the $B_{2}$ case our results imply the quantum integrability of the system with Hamiltonian

$$
H=-\triangle+F(x)+F(y)+G(x+y)+G(x-y),
$$

where $F=f^{\prime}-f^{2}, G=g^{\prime}-g^{2}$, and $f, g$, are given by the formula (14). The commuting operator $K$ has the form $\operatorname{Res}\left(\nabla_{1}^{2} \nabla_{2}^{2}\right)$, where $\nabla_{i}$ are the corresponding generalized Dunkl operators (6). The quantum integrability of this systems was independently established in [19].

It would be interesting to understand the relation between this construction and the recent construction of elliptic Dunkl operators of [20], which are formal infinite linear combinations of affine Weyl group reflections.

Acknowledgments. Two of us (V. B. and A. V.) are grateful to the University of Maryland at College Park and especially to Prof. S. P. Novikov for the hospitality during February 1994, when this work was completed. G. F. is grateful to IHES, where part of this work was done, for hospitality, and thanks V. Pasquier for explanations and discussions. 


\section{Appendix: Properties of the function $\sigma_{\lambda}(z)$}

We give some properties of the function $\sigma_{\lambda}(z)$ (29). This function can be expressed in terms of the Weierstrass functions (see, e.g., [11]) with periods $1, \tau$ :

$$
\sigma_{\lambda}(z)=\frac{\sigma(z-\lambda)}{\sigma(z) \sigma(-\lambda)} e^{2 \eta_{1} z \lambda}, \quad \eta_{1}=\zeta\left(\frac{1}{2}\right) .
$$

\section{Proposition 19 (i) $\sigma_{\lambda}(z)=-\sigma_{-\lambda}(-z)$,}

(ii) $\sigma_{\lambda}(z)=-\sigma_{z}(\lambda)$,

(iii) $\sigma_{\lambda}(z) \sigma_{\mu}(w)-\sigma_{\lambda+\mu}(w) \sigma_{\lambda}(z-w)-\sigma_{\mu}(w-z) \sigma_{\lambda+\mu}(z)=0$,

(iv) $\sigma_{\lambda}(z) \sigma_{-\lambda}(z)=\wp(z)-\wp(\lambda)$,

(v) $\lim _{\lambda \rightarrow 0} \sigma_{\lambda}^{\prime}(z)=-\wp(z)-2 \eta_{1}$.

Proof: (i) and (ii) are obvious. The functional equation (iii) follows from the fact the left hand side is 1-periodic in, say, $z$, and is multiplied by $\exp (2 \pi i \lambda)$ as $z \rightarrow z+\tau$. Moreover, the singularity as $z \rightarrow 0$ (and thus as $z \rightarrow m+n \tau$, $n, m \in \mathbf{Z}$, by quasiperiodicity) is removable since $\sigma_{\lambda}(z)=1 / z+O(1)$. Thus we have an entire 1-periodic function of $z$ with non-trivial multipliers as $z \rightarrow z+\tau$. Such a function must vanish.

(iv) The left hand side is an even elliptic function with double pole with unit coefficient at the origin, and no other pole in the period parallelogram. Thus it is of the form $\wp(z)+\psi(\lambda)$, for some function $\psi$. Then use $\sigma_{\lambda}(\lambda)=0$ to determine $\psi$.

(v) Recall that $\sigma^{\prime}(z)=\zeta(z) \sigma(z), \zeta^{\prime}(z)=-\wp(z)$ and $\sigma(z)=z+O\left(z^{5}\right)$. We have

$$
\begin{aligned}
\frac{d}{d z} \frac{\sigma(z-\lambda)}{\sigma(z) \sigma(-\lambda)} & =\frac{\sigma(z-\lambda)(\zeta(z-\lambda)-\zeta(z))}{\sigma(z) \sigma(-\lambda)} \\
& =\zeta^{\prime}(z)+O(\lambda) .
\end{aligned}
$$

Thus $\sigma_{\lambda}^{\prime}(z)=-\wp(z)+O(\lambda)+2 \eta_{1} \lambda \sigma_{\lambda}(z)$, but $\lambda \sigma_{\lambda}(z) \rightarrow-1$ as $\lambda$ tends to 0 .

Remark. Note that the functional equation (iii) is a special case $\left(A_{2}\right)$ of Theorem 12. 


\section{References}

[1] C. F. Dunkl, Differential-difference operators associated to reflection groups, Trans. Math. Math. Soc. 311, 167-183 (1989)

[2] A. P. Veselov, Calogero quantum problem, KZ equation and Huygens principle, to appear in Theor. Math. Phys. 98, n. 3, 524-535 (1994)

[3] I. V. Cherednik, A unification of KZ and Dunkl operators via affine Hecke algebras. Invent. Math. 106, 411-431 (1991)

[4] V. M. Buchstaber and A. P. Veselov, Dunkl operators, functional equations and transformations of elliptic genera, Uspekhi Mat. Nauk 49, n. 2 (1994)

[5] V. M. Buchstaber, Functional equations associated with addition theorems for elliptic functions and two-valued algebraic groups, Russ. Math. Surv. 45, 213-244 (1990) (Russian)

[6] V. M. Buchstaber, MPI report, Bonn, 1992

[7] Y. Shibukawa and K. Ueno, Completely $\mathbf{Z}$ symmetric $R$-matrix, Waseda University preprint, 1992

[8] G. Felder and V. Pasquier, A simple construction of elliptic $R$-matrices, hep-th/9402011, to appear in Lett. Math. Phys.

[9] G. J. Heckman, A remark on the Dunkl differential-difference operators, in W. Barker and P. Sally (eds.) "Harmonic analysis on reductive groups", Birkhäuser, Basel, 1991

[10] N. Bourbaki, Groupes et algèbres de Lie, Chap. VI, Masson, 1981

[11] E. T. Whittaker and G. N. Watson, Modern analysis, Cambridge University Press, 1927

[12] J. Moser, Three integrable Hamiltonian systems connected with isospectral deformations, Adv. Math. 16, 441-416

[13] I. M. Krichever, Elliptic solutions of the KP equation and integrable systems of particles, Fun. Anal. Appl. 14, 282-290 (1980) 
[14] M. A. Olshanetsky and A. M. Perelomov, Classical integrable finitedimensional systems related to Lie algebras, Phys. Rep. 71, 313-400 (1981)

[15] M. Bruschi and F. Calogero, General analytic solution of certain functional equations of addition type, SIAM J. Math. Anal. 21, 1019-1030

[16] A. A. Belavin, Dynamical symmetry of integrable quantum systems, Nucl. Phys. B180 [FS2], 189-200 (1981)

[17] O. Chalykh, A. P. Veselov, Commutative partial differential operators and Lie algebras, Preprint of FIM (ETH,Zürich), 1988, Commun. Math. Phys., 126, 597-611 (1990)

[18] G. Heckman, An elementary approach to the hypergeometric shift operators of Opdam, Inv. Math. 103, 341-350 (1991).

[19] H. Ochiai, T. Oshima, H. Sekiguchi, Commuting families of symmetric differential operators, to appear in Proc. Japan Acad. 70A, 62-66 (1994)

[20] I. Cherednik, Elliptic quantum many-body problem and affine Knizhnik-Zamolodchikov equation, UNC preprint, hep-th/940316, 1994

[21] M. A. Olshanetsky, A.M. Perelomov, Quantum integrable systems related to Lie algebras, Phys. Rep. 94, N6, 313-404 (1983) 